\title{
Utilização do Acesso Radial Elimina a Ocorrência de Sangramento Grave Relacionado ao Sítio de Punção após Intervenção Coronária Percutânea Primária
}

\author{
Pedro Beraldo de Andrade ${ }^{1,2}$, Marden André Tebet ${ }^{1,2}$, Felipe Souza Maia da Silva ${ }^{2}$, \\ Mônica Vieira Athanazio de Andrade', Luiz Alberto Mattos ${ }^{1,3}$, André Labrunie ${ }^{1,2}$
}

\section{RESUMO}

Introdução: $O$ acesso radial associa-se a baixa ocorrência de complicações vasculares. Essa característica o torna vantajoso nas síndromes coronárias agudas, em que é frequente o uso agressivo de terapias antiplaquetária e antitrombótica. O objetivo do estudo foi avaliar a eficácia e a segurança do acesso radial na realização de intervenção percutânea primária, em que atrasos na reperfusão advindos de maior dificuldade técnica representariam um fator limitante. Método: Registro envolvendo pacientes submetidos a intervenção percutânea primária pelo acesso radial. Os desfechos de eficácia constituíram-se em mortalidade hospitalar, tempo porta-balão, obtenção de fluxo final TIMI III e conclusão do procedimento sem troca da via de acesso. A segurança foi avaliada por meio de taxa de sangramento grave e ocorrência de complicações relacionadas ao sítio de punção. Resultados: Entre fevereiro de 2007 e novembro de 2009, 211 pacientes foram submetidos a intervenção percutânea primária, 201 por meio de acesso radial. A média de idade foi de 59 anos, sendo $27,4 \%$ do sexo feminino e $22,4 \%$ portadores de diabetes melito. Foram obtidos fluxo final TIMI III em 91,5\% dos pacientes e resolução do segmento ST > 50\% em $88,5 \%$, com taxa de crossover de 1,5\%. A mortalidade hospitalar foi de 4,5\% e a taxa de sangramento grave foi de $1 \%$, sendo um episódio de hemorragia digestiva alta e uma queda de hemoglobina de $5 \mathrm{~g} / \mathrm{dl}$. As complicações vasculares relacionadas ao sítio de punção limitaram-se a três casos de hematoma tipo II e a dois casos de hematoma tipo III, sem repercussão clínica e com resolução espontânea. Conclusão: O acesso radial representa uma via eficaz e segura para

\section{ABSTRACT}

\section{Transradial Approach Eliminates Puncture Site Major Bleeding After Primary Percutaneous Coronary Intervention}

Background: Transradial approach is associated with a low incidence of vascular complications. This peculiarity makes it useful in acute coronary syndromes, where aggressive antiplatelet and antithrombotic therapy is frequently used. The aim of this study was to evaluate the efficacy and safety of transradial approach in primary percutaneous intervention, where delays in achieving reperfusion resulting from technical difficulties are unacceptable. Method: Registry including consecutive patients undergoing primary percutaneous intervention by transradial approach. The efficacy endpoints were in-hospital mortality, door-to-balloon time, final TIMI III flow and access site crossover. Safety was assessed by the rate of major bleeding and complications related to the puncture site. Results: From February 2007 to November 2009, 211 patients underwent primary percutaneous intervention, 201 of them by transradial approach. Mean age was 59 years, $27.4 \%$ were female and $22.4 \%$ had diabetes mellitus. Final TIMI III flow was achieved in $91.5 \%$ of patients, ST segment resolution $>50 \%$ in $88.5 \%$, with a crossover rate of $1.5 \%$. In-hospital mortality was $4.5 \%$ and the rate of major bleeding was $1 \%$, with one episode of upper gastrointestinal bleeding and a decrease in hemoglobin $>5 \mathrm{~g} / \mathrm{dl}$. Vascular complications related to the puncture site were limited to 3 cases of type II hematoma and 2 cases of type III hematoma, with no clinical impact and spontaneous resolution. Conclusion: The transradial approach,

1 Irmandade da Santa Casa de Misericórdia de Marília - Marília, SP, Brasil.

2 Hospital do Coração de Londrina - Londrina, PR, Brasil.

3 Instituto Dante Pazzanese de Cardiologia - São Paulo, SP, Brasil. Correspondência: Pedro Beraldo de Andrade. Av. Vicente Ferreira, 828 - Marília, SP, Brasil - CEP 17515-900

E-mail: pedroberaldo@gmail.com

Recebido em: 11/10/2010 • Aceito em: 2/12/2010 
a realização de intervenção coronária percutânea primária por operadores habituados ao emprego da técnica, com ocorrência virtualmente nula de complicações vasculares graves relacionadas ao sítio de punção.

DESCRITORES: Artéria radial. Angioplastia transluminal percutânea coronária. Hemorragia.

D eambulação precoce, maior conforto ao paciente, redução do tempo de hospitalização, redução de custos e menor taxa de complicações vasculares relacionadas ao sítio de punção são atributos inerentes à técnica radial. ${ }^{1}$ Em revisão sistemática e meta-análise de estudos randomizados, o acesso radial reduziu em $73 \%$ a prevalência de sangramento grave quando comparado ao acesso femoral em procedimentos coronários invasivos diagnósticos e terapêuticos $(0,05 \%$ vs. 2,3\%; P $<0,001)$, com tendência a redução de morte, infarto agudo do miocárdio ou acidente vascular encefálico. ${ }^{2}$

Essas características tornam a adoção do acesso radial potencialmente vantajosa na realização de intervenção coronária percutânea no cenário das síndromes isquêmicas agudas, particularmente no infarto agudo do miocárdio com supradesnivelamento do segmento ST, em que é frequente o uso agressivo de terapias antiplaquetária e antitrombótica. Porém, as evidências atuais imputam à técnica radial maior necessidade de troca da via de acesso (crossover), bem como maior inabilidade em ultrapassar a lesão com o guia, balão ou stent durante o procedimento.

Atrasos para a efetivação com sucesso da reperfusão coronária advindos de maior dificuldade relacionada à obtenção da via de acesso ou adequado suporte para o avanço de instrumentais são preocupações pertinentes que promovem a análise crítica da utilização da técnica radial nesse contexto. O tempo para a reperfusão correlaciona-se com a extensão do infarto e a área de salvamento miocárdico ${ }^{3}$, fatores determinantes e de grande impacto na morbidade, na mortalidade e no prognóstico. ${ }^{4}$ Assim, o presente estudo objetiva avaliar a segurança e a eficácia do acesso radial na realização de intervenção coronária percutânea primária em uma população não-selecionada representativa da prática diária, em um centro que prioriza a utilização dessa via na execução de procedimentos coronários invasivos.

\section{MÉTODOS}

Pacientes consecutivos com diagnóstico de infarto agudo do miocárdio com supradesnivelamento do segmento ST nas primeiras 12 horas de evolução, submetidos a intervenção coronária percutânea primária pelo acesso radial, foram incluídos em um registro prospectivo de eficácia e segurança. Os desfe- performed by operators familiarized with the technique, is a safe and effective alternative for primary percutaneous coronary intervention, with virtually no major vascular complications related to the puncture site.

KEY-WORDS: Radial artery. Angioplasty, transluminal, percutaneous coronary. Hemorrhage.

chos de eficácia constituíram-se em mortalidade hospitalar, reinfarto, acidente vascular encefálico, tempo porta-balão, porcentual do diâmetro de estenose da lesão-alvo $<30 \%$ associado a fluxo final TIMI III, resolução do supradesnível do segmento ST $\geq 50 \%$ e efetivação do procedimento sem necessidade de troca da via de acesso. Tempos de procedimento e fluoroscopia foram obtidos a partir do início da punção arterial até a retirada do introdutor. A segurança foi avaliada por meio da ocorrência de sangramento grave e complicações vasculares relacionadas ao sítio de punção.

Sangramento grave foi definido como hemorragia intracraniana, intraocular ou retroperitoneal, queda de hemoglobina superior a $3 \mathrm{~g} / \mathrm{dl}$ ou necessidade de transfusão sanguínea utilizando-se dois ou mais concentrados de hemácias. Os hematomas foram graduados de acordo com a classificação do estudo Early Discharge after Transradial Stenting of Coronary Arteries (EASY) ${ }^{5}$ : tipo I, $\leq 5 \mathrm{~cm}$ de diâmetro; tipo II, $\leq 10 \mathrm{~cm}$ de diâmetro; tipo III, > $10 \mathrm{~cm}$, sem atingir o cotovelo; tipo IV, hematoma estendendo-se além do cotovelo; tipo $\mathrm{V}$, qualquer hematoma com injúria isquêmica à mão. Complicações relacionadas ao sítio de punção além de hematomas incluíram fístula arteriovenosa, pseudoaneurisma, oclusão arterial assintomática, necessidade de reparo vascular cirúrgico e infecção local.

Por meio de hiperextensão do punho e infiltração de 1-2 $\mathrm{ml}$ de xilocaína a $2 \%$, puncionava-se a artéria radial $1 \mathrm{~cm}$ proximal ao processo estiloide do rádio, utilizando-se agulha com cateter de polietileno tipo Jelco ${ }^{\mathrm{TM}}$ calibres 20-22 e técnica de Seldinger. Após a punção, introduzia-se um fio-guia de 0,021 polegada, seguido de pequena incisão cutânea com lâmina de bisturi no 11 e inserção de introdutor curto $(<11 \mathrm{~cm})$ 5-7 F. Administrava-se uma solução contendo 5.000 UI de sulfato de heparina e $10 \mathrm{mg}$ de mononitrato de isossorbida através da extensão do introdutor. Ao término do procedimento, removia-se imediatamente o introdutor e obtinha-se hemostasia com curativo compressivo através de bandagem elástica adesiva porosa (Tensoplast ${ }^{\mathrm{TM}}$ ) ou pulseira compressora seletiva (TR Band ${ }^{\mathrm{TM}}$, Terumo Medical Corporation, Tóquio, Japão). Procedia-se a exame clínico do sítio de punção e avaliação do pulso radial no momento da alta hospitalar.

As variáveis categóricas foram expressas em frequência e porcentagem e as variáveis contínuas foram expressas em média e desvio padrão. 
Andrade PB, et al. Utilização do Acesso Radial Elimina a Ocorrência de Sangramento Grave Relacionado ao Sítio de Punção após Intervenção Coronária Percutânea Primária. Rev Bras Cardiol Invasiva. 2010;18(4):387-91.

\section{RESULTADOS}

Entre fevereiro de 2007 e novembro de 2009, 211 pacientes foram submetidos a intervenção coronária percutânea primária, dos quais 201 (95,3\%) por meio de acesso radial, compondo a amostra avaliada, 9 (4,3\%) pelo acesso femoral e $1(0,4 \%)$ pelo acesso ulnar. Dentre as circunstâncias que motivaram o emprego da técnica femoral prevaleceram histórico de cirurgia de revascularização miocárdica prévia (44\%), quadro de choque cardiogênico sem pulso radial palpável (33\%) e insuficiência renal crônica dialítica com fístula arteriovenosa em membro superior (23\%).

Do total de pacientes avaliados, $140(69,7 \%)$ foram inicialmente socorridos em hospitais secundários ou unidades primárias de atendimento e transferidos, após confirmação eletrocardiográfica do diagnóstico, pelo Serviço de Atendimento Móvel de Urgência (SAMU), sendo o tempo médio de transferência de $40 \pm 27$ minutos e o tempo de isquemia, de $4 \mathrm{~h} 39 \mathrm{~min} \pm 2 \mathrm{~h} 33 \mathrm{~min}$. As características clínicas dos pacientes estão expressas na Tabela 1. A média de idade foi de 59,6 anos, sendo $27,4 \%$ pertencentes ao sexo feminino e $22,4 \%$, portadores de diabetes melito.

A farmacoterapia antiplaquetária utilizada constituiu-se, na totalidade dos casos, em ácido acetilsalicílico 300 mg, seguido de dose de manutenção de 100 mg, clopidogrel $300 \mathrm{mg}$ a $600 \mathrm{mg}$, seguido de dose de manutenção de $75 \mathrm{mg}$, e inibidor de glicoproteína IIb/IIla tirofibana em $145(72,1 \%)$ procedimentos. Foi administrada dose de ataque intracoronária de tirofibana em $23 \%$ desses pacientes, seguida de dose de manutenção por via intravenosa; nos restantes $77 \%$, as doses de ataque e de manutenção foram administradas por via intravenosa. $\mathrm{O}$ agente antitrombótico de escolha foi a heparina não-fracionada, na dose de $100 \mathrm{U} / \mathrm{kg}$ intravenosa. Cateteres de aspiração manual de trombo foram empregados em $95(47,3 \%)$ casos, preferencial-

TABELA 1

\section{Características clínicas basais}

\begin{tabular}{lc}
\hline Variáveis & $\mathbf{n}=\mathbf{2 0 1}$ \\
\hline Sexo feminino, n (\%) & $55(27,4)$ \\
Idade, anos & $59,6 \pm 11,5$ \\
Hipertensão arterial sistêmica, n (\%) & $144(71,6)$ \\
Diabetes melito, n (\%) & $45(22,4)$ \\
Dislipidemia, n (\%) & $51(25,4)$ \\
Tabagismo, n (\%) & $90(44,8)$ \\
Infarto do miocárdio prévio, n (\%) & $11(5,5)$ \\
Intervenção coronária & $9(4,5)$ \\
percutânea prévia, n (\%) & $5(2,5)$ \\
Revascularização miocárdica prévia, n $(\%)$ & $19(9,5)$ \\
Classe Killip III/IV, n (\%) & \\
\hline $\mathrm{n}=$ número de pacientes. & \\
\hline
\end{tabular}

mente naqueles com escore de trombo $\mathrm{TIMI}^{6}$ graduado entre 3-5.

As características dos procedimentos estão expressas na Tabela 2. Diâmetro de estenose residual inferior a 30\% com fluxo final TIMI III foi alcançado em 184 $(91,5 \%)$ pacientes, enquanto a resolução do supradesnivelamento do segmento ST $\geq 50 \%$ foi observada em $178(88,5 \%)$ dos pacientes. A taxa de crossover situou-se em $1,5 \%$, pela necessidade de troca da via de acesso inicialmente selecionada em três casos, decorrente de apoio insuficiente para a conclusão do procedimento, presença de tortuosidade radioulnar que impedia a progressão dos cateteres, e oclusão da artéria braquial direita após coronariografia eletiva prévia pela técnica de Sones. Apenas no primeiro caso o maior grau de dificuldade técnica associou-se a tempo porta-balão superior a 90 minutos.

Durante a internação, 9 pacientes $(4,5 \%)$ evoluíram para óbito, $5(55 \%)$ dos quais em decorrência de choque cardiogênico. Trombose subaguda de stent, encefalopatia anóxica secundária a parada cardiorrespiratória, fibrilação ventricular refratária e edema agudo de pulmão responderam cada qual por um óbito hospitalar. Dois pacientes apresentaram episódio de sangramento grave, sendo um por hemorragia digestiva alta com necessidade de transfusão de duas unidades de concentrado de hemácias e o outro por queda de hemoglobina de $5 \mathrm{~g} / \mathrm{dl}$, sem exteriorização clínica. As complicações vasculares relacionadas ao sítio de punção limitaram-se a três casos de hematoma tipo II e a dois casos de hematoma tipo III, sem repercussão clínica e com resolução espontânea (Tabela 3).

TABELA 2

Características angiográficas e do procedimento

\begin{tabular}{lc}
\hline Variáveis & $\mathbf{n}=\mathbf{2 0 1}$ \\
\hline Tempo de isquemia, horas & $4,39 \pm 2,33$ \\
Tempo porta-balão, minutos & $81,8 \pm 26,9$ \\
Duração do procedimento, minutos & $47,1 \pm 21,1$ \\
Tempo de fluoroscopia, minutos & $12,1 \pm 6,6$ \\
Taxa de crossover, n (\%) & $3(1,5)$ \\
Vaso-alvo, n (\%) & \\
Descendente anterior & $85(42,3)$ \\
Coronária direita & $85(42,3)$ \\
Circunflexa & $31(15,4)$ \\
Lesão tipo B2/C, n (\%) & $178(88,6)$ \\
Implante de stent, $\mathrm{n}(\%)$ & $196(97,5)$ \\
Calibre do introdutor, $\mathrm{n}(\%)$ & \\
5 F & $2(1)$ \\
6 F & $198(98,5)$ \\
7 F & $1(0,5)$ \\
Balão intra-aórtico, $\mathrm{n}(\%)$ & $4(2)$ \\
\hline
\end{tabular}

$\mathrm{n}=$ número de pacientes. 
TABELA 3

Desfechos hospitalares de eficácia e segurança

\begin{tabular}{lc} 
Variáveis & $\mathbf{n}=\mathbf{2 0 1}$ \\
\hline Fluxo epicárdico final TIMI III, n (\%) & $184(91,5)$ \\
Resolução do segmento ST $\geq 50 \%, \mathrm{n}(\%)$ & $178(88,5)$ \\
Mortalidade, n (\%) & $9(4,5)$ \\
Acidente vascular encefálico, n (\%) & 0 \\
Reinfarto, n (\%) & $3(1,5)$ \\
Revascularização miocárdica & 0 \\
de urgência, n (\%) & \\
Trombose do stent aguda e subaguda, n $(\%)$ & $3(1,5)$ \\
Sangramento grave, n (\%) & $2(1)$ \\
Complicações vasculares no & $5(2,5)$ \\
sítio de punção, n (\%) & \\
\hline $\mathrm{n}=$ número de pacientes. & \\
\hline
\end{tabular}

\section{DISCUSSÃO}

O primeiro relato da realização de intervenção coronária percutânea primária com sucesso pelo acesso radial é de 1996, efetivada em 2 pacientes com contraindicação para utilização do acesso femoral. ${ }^{7}$ Com a maior aceitação e propagação de seu uso, diferentes centros expuseram suas experiências iniciais com a utilização da técnica no tratamento de pacientes com infarto agudo do miocárdio. ${ }^{8-10} \mathrm{Em}$ comum, os resultados desse procedimento exibem elevadas taxas de sucesso e baixa incidência de complicações vasculares, quando realizado por operadores experientes e envolvendo pacientes selecionados.

Posteriormente, registros consecutivos e controlados comparando os acessos radial e femoral no tratamento do infarto agudo do miocárdio com supradesnivelamento do segmento ST, em população representativa do mundo real, demonstraram que a utilização do primeiro não promoveu diferenças no tempo de reperfusão, bem como no sucesso e na duração total do procedimento. ${ }^{11,12}$ Com relação aos desfechos adversos, os resultados apresentados não apontaram diferenças em eventos cardiovasculares graves, ao passo que a taxa de complicações hemorrágicas relacionadas ao sítio de punção foi nula entre os pacientes que utilizaram o acesso radial e variou de 1,5\% a $8,5 \%$ entre aqueles que utilizaram o acesso femoral, corroborando os achados de nossa casuística.

Diante da administração sistemática do inibidor de glicoproteína Ilb/IIla abciximab em pacientes submetidos a intervenção coronária percutânea primária, Philippe et al. ${ }^{13}$ demonstraram que a técnica radial promoveu redução significante de sangramentos graves $(0 \%$ vs. $5,5 \%$; $P=0,03)$ e do tempo de permanência hospitalar (4,5 dias vs. 5,9 dias; $\mathrm{P}=0,05)$, quando comparada à técnica femoral. De fato, a segurança proporcionada pelo acesso radial na redução de complicações vasculares justifica a elevada utilização adjunta de inibidor de glicoproteína IIb/IIla observada em nossa rotina de atendimento ao paciente infartado ${ }^{14}$, amparada por recomendações recentes de diretrizes ${ }^{15}$, mas de pequena observância em centros nos quais prevalece o acesso femoral.

Em recente meta-análise de 12 estudos envolvendo 3.324 pacientes submetidos a intervenção coronária percutânea primária ou de resgate, que compara os acessos femoral e radial, o acesso radial demonstrou redução significante de $70 \%$ do risco de sangramento grave $(0,8 \%$ vs. $2,6 \% ; P=0,0001)$, bem como de morte, infarto agudo do miocárdio ou acidente vascular encefálico $(3,7 \%$ vs. $6,6 \% ; P=0,01)$ e tempo de hospitalização. ${ }^{16}$ Não se observou diferença na duração total do procedimento e no tempo de reperfusão, embora o acesso radial tenha se associado a maior duração de fluoroscopia e maior necessidade de crossover. A mortalidade hospitalar de 4,5\% observada em nosso estudo é compatível com o tempo porta-balão médio de 82 minutos, conforme publicação do American College of Cardiology National Cardiovascular Data Registry17, segundo a qual 43.801 pacientes submetidos a intervenção coronária percutânea primária entre 2005 e 2006, com tempo portabalão médio de 83 minutos, exibiram mortalidade hospitalar de $4,6 \%$.

Em nossa experiência, a utilização rotineira do acesso radial em intervenção coronária percutânea primária mostrou-se factível, traduzida pela elevada taxa de sucesso do procedimento e baixo índice de crossover. Pristipino et al. ${ }^{18}$ demonstraram, no Prospective Registry of Vascular Access in Interventions in Lazio region (PREVAIL), correlação inversa entre a frequência de utilização do acesso radial pelo operador e a necessidade de troca da via de acesso, situando-se em até $33 \%$ entre aqueles que a empregam em menos de $25 \%$ dos casos, reduzindo-se para $3 \%$ entre os operadores cujo porcentual é superior a $85 \%$.

Assim, o perfil de eficácia e segurança associado ao acesso radial na realização de intervenção coronária percutânea primária torna sua escolha recomendável, desde que efetivada por operadores habilitados e familiarizados com seu emprego, sobretudo aqueles que elegeram a técnica como sua primeira opção na realização de procedimentos coronários invasivos, suplantando assim a curva de aprendizagem ${ }^{19,20}$ e eventuais dificuldades técnicas, permitindo a rápida e desejada conclusão do procedimento.

\section{Limitações do estudo}

O presente estudo possui como limitações sua natureza observacional, a ausência de um grupo comparativo de pacientes submetidos a intervenção coronária percutânea primária pelo acesso femoral, ser conduzido em um único centro, caracterizado por 
Andrade PB, et al. Utilização do Acesso Radial Elimina a Ocorrência de Sangramento Grave Relacionado ao Sítio de Punção após Intervenção Coronária Percutânea Primária. Rev Bras Cardiol Invasiva. 2010;18(4):387-91.

operadores familiarizados com a técnica radial, e ausência de seguimento clínico tardio.

\section{CONCLUSÕES}

Os resultados obtidos atestam que a utilização rotineira do acesso radial na intervenção coronária percutânea primária, quando empregado por operadores familiarizados com a técnica, é uma estratégia eficaz, associada a elevado índice de sucesso do procedimento, não implicando retardos a sua efetivação, e segura, eliminando o risco de sangramento grave decorrente de complicações relacionadas à via de acesso.

\section{CONFLITO DE INTERESSES}

Os autores declararam inexistência de conflito de interesses relacionado a este manuscrito.

\section{REFERÊNCIAS}

1. Agostoni $\mathrm{P}$, Biondi-Zoccai GG, de Benedictis $\mathrm{ML}$, Rigattieri S, Turri M, Anselmi M, et al. Radial versus femoral approach for percutaneous coronary diagnostic and interventional procedures; systematic overview and meta-analysis of randomized trials. J Am Coll Cardiol. 2004;44(2):349-56.

2. Jolly SS, Amlani S, Hamon M, Yusuf S, Mehta SR. Radial versus femoral access for coronary angiography or intervention and the impact on major bleeding and ischemic events: a systematic review and meta-analysis of randomized trials. Am Heart J. 2009;157(1):132-40.

3. Francone M, Ducci CB, Carbone I, Canali E, Scardala R, Calabrese FA, et al. Impact of primary coronary angioplasty delay on myocardial salvage, infarct size, and microvascular damage in patients with ST-segment elevation myocardial infarction. J Am Coll Cardiol. 2009;54(23):2145-53.

4. De Luca G, Suryapranata H, Ottervanger JP, Antman EM. Time delay to treatment and mortality in primary angioplasty for acute myocardial infarction: every minute of delay counts. Circulation. 2004;109(10):1223-5.

5. Bertrand OF, De Larochellière R, Cabau JR, Proulx G, Gleeton $\mathrm{O}$, Nguyen $\mathrm{CM}$, et al. A randomized study comparing same-day home discharge and abciximab bolus only to overnight hospitalization and abciximab bolus and infusion after transradial coronary stent implantation. Circulation. 2006; 114(24):2636-43.

6. Gibson CM, Lemos JA, Murphy SA, Marble SJ, McCabe $\mathrm{CH}$, Cannon $\mathrm{CP}$, et al. Combination therapy with abciximab reduces angiographically evident thrombus in acute myocardial infarction: a TIMI 14 substudy. Circulation. 2001;103(21):2550-4.

7. Steg G, Aubry P. Radial access for primary PTCA in patients with acute myocardial infarction and contraindication to or impossible femoral access. Cathet Cardiovasc Diagn. 1996; 39(4):424-6.
8. Mathias DW, Bigler L. Transradial coronary angioplasty and stent implantation in acute myocardial infarction: initial experience. J Invasive Cardiol. 2000;12(11):547-9.

9. Mulukutla SR, Cohen HA. Feasibility and efficacy of transradial access for coronary interventions in patients with acute myocardial infarction. Catheter Cardiovasc Interv. 2002;57(2): 167-71.

10. Salles JAB, Andrea JCM, Cortes LA, Carestiato LV, Santos LFCS, Figueira HR. Análise comparativa de segurança e eficácia entre as vias de acesso radial e femoral na realização de intervenção coronária percutânea no infarto agudo do miocárdio. Rev Bras Cardiol Invasiva. 2009;17(4):498-504.

11. Louvard $Y$, Ludwig J, Lefèvre $T$, Schmeisser $A$, Brück $M$, Scheinert D, et al. Transradial approach for coronary angioplasty in the setting of acute myocardial infarction: a dual-center registry. Catheter Cardiovasc Interv. 2002;55(2):206-11.

12. Ziakas A, Klinke $P$, Mildenberger $R$, Fretz $E$, Williams $M$, Della Siega $A$, et al. Comparison of the radial and femoral approaches in percutaneous coronary intervention for acute myocardial infarction. Am J Cardiol. 2003;91(5):598-600.

13. Philippe F, Larrazet F, Meziane $T$, Dibie A. Comparison of transradial vs. transfemoral approach in the treatment of acute myocardial infarction with primary angioplasty and abciximab. Catheter Cardiovasc Interv. 2004;61(1):67-73.

14. Tebet MA, Andrade PB, Gentile M, Mattos LA, Labrunie A. Angioplastia primária via radial com doses aumentadas de tirofiban: uma técnica segura e efetiva. Rev Bras Cardiol Invasiva. 2007;15(3):255-60.

15. Wijns W, Kolh P, Danchin N, Di Mario C, Falk V, Folliguet $\mathrm{T}$, et al. Guidelines on myocardial revascularization. The Task Force on Myocardial Revascularization of the European Society of Cardiology (ESC) and European Association for Cardio-Thoracic Surgery (EACTS). Eur Heart J. 2010;31(2): 2501-55.

16. Vorobcsuk A, Kónyi A, Aradi D, Horváth IG, Ungi I, Louvard $Y$, et al. Transradial versus transfemoral percutaneous coronary intervention in acute myocardial infarction: systematic overview and meta-analysis. Am Heart J. 2009;158(5):814-21.

17. Rathore SS, Curtis JP, Chen J, Wang Y, Nallamothu BK, Epstein AJ, et al. Association of door-to-balloon time and mortality in patients admitted to hospital with ST elevation myocardial infarction: national cohort study. BMJ. 2009; 338:b1807.

18. Pristipino C, Trani C, Nazzaro MS, Berni A, Patti G, Patrizi $R$, et al. Major improvement of percutaneous cardiovascular procedures outcomes with radial artery catheterization. Results from the PREVAIL study. Heart. 2009;95(6):476-82.

19. Goldberg SL, Renslo R, Sinow R, French WJ. Learning curve in the use of the radial artery as vascular access in the performance of percutaneous transluminal coronary angioplasty. Cathet Cardiovasc Diagn. 1998;44(2):147-52.

20. Labrunie A, Tebet MA, Andrade PB, Andrade MVA, Conterno LO, Mattos LAP, et al. Coronariografia via transradial: curva de aprendizagem, avaliada por estudo multicêntrico. Rev Bras Cardiol Invasiva. 2009;17(1):82-7. 